\title{
Parallax-sensitive remapping of visual space in occipito-parietal alpha-band activity during whole-body motion
}

\author{
T. P. Gutteling, L. P. J. Selen, and W. P. Medendorp \\ Radboud University Nijmegen, Donders Institute for Brain, Cognition and Behaviour, Nijmegen, The Netherlands
}

Submitted 30 June 2014; accepted in final form 10 December 2014

Gutteling TP, Selen LP, Medendorp WP. Parallax-sensitive remapping of visual space in occipito-parietal alpha-band activity during whole-body motion. J Neurophysiol 113: 1574-1584, 2015. First published December 10, 2014; doi:10.1152/jn.00477.2014.Despite the constantly changing retinal image due to eye, head, and body movements, we are able to maintain a stable representation of the visual environment. Various studies on retinal image shifts caused by saccades have suggested that occipital and parietal areas correct for these perturbations by a gaze-centered remapping of the neural image. However, such a uniform, rotational, remapping mechanism cannot work during translations when objects shift on the retina in a more complex, depth-dependent fashion due to motion parallax. Here we tested whether the brain's activity patterns show parallax-sensitive remapping of remembered visual space during whole-body motion. Under continuous recording of electroencephalography (EEG), we passively translated human subjects while they had to remember the location of a world-fixed visual target, briefly presented in front of or behind the eyes' fixation point prior to the motion. Using a psychometric approach we assessed the quality of the memory update, which had to be made based on vestibular feedback and other extraretinal motion cues. All subjects showed a variable amount of parallaxsensitive updating errors, i.e., the direction of the errors depended on the depth of the target relative to fixation. The EEG recordings show a neural correlate of this parallax-sensitive remapping in the alphaband power at occipito-parietal electrodes. At parietal electrodes, the strength of these alpha-band modulations correlated significantly with updating performance. These results suggest that alpha-band oscillatory activity reflects the time-varying updating of gaze-centered spatial information during parallax-sensitive remapping during wholebody motion.

spatial updating; EEG; alpha power; whole-body motion; remapping

WE PERCEIVE THE WORLD as a stable reality, despite the ubiquitous changes in visual input due to our own movements. We do not perceive a visual shift when we make a saccade, even though the image of the world moves briskly on our retinas (Bridgeman and Nardello 1994; Schlag and Schlag-Rey 2002; von Helmholtz 1867). Also, when we walk around, our perception seems not to be disturbed by the even more complex changes in the optic flow on our retinas (see Angelaki and Hess 2005 for review). How does our brain create this percept of visual stability?

There is evidence that the brain codes dynamic visual representations of the environment, which are remapped in gaze-centered coordinates when the eyes move. Various cortical and subcortical regions have been implicated in this remapping during saccadic eye movements in both primates (Colby and Goldberg 1999; Duhamel et al. 1992) and humans (Belle-

Address for reprint requests and other correspondence: T. P. Gutteling, Montessorilaan 3, 6525 HR Nijmegen, The Netherlands (e-mail: t.gutteling @ donders.ru.nl). baum and Daum 2006; Medendorp et al. 2003a; Merriam et al. 2003; Morris et al. 2007). Behavioral studies and preliminary neurophysiological reports have also provided evidence for gaze remapping during smooth pursuit eye movements (Blohm et al. 2003, 2005; Dash et al. 2012). Do such mechanisms, involved in saccadic or smooth pursuit remapping with the head immobilized, also operate to enable visual stability during head and body motion, taking into account vestibular and other signals? When the body is brought into motion, as when driving a car, vestibular feedback informs the brain about the motion (Klier et al. 2008; $\mathrm{Li}$ et al. 2005; Li and Angelaki 2005), but how these signals contribute to visual remapping has not been revealed.

In geometric terms, when the body translates through space, world-stationary objects move at different speeds and in different directions relative to the retina, depending on their distance from the eyes' fixation point (Van Pelt and Medendorp 2007). This geometric property, called motion parallax, does not play a role in visual remapping during saccades but must be taken into account by a gaze-centered remapping mechanism during body translations. Do the neural mechanisms for visual stability show this level of sophistication, or does the brain resort to a different gaze-independent mechanism?

Recent behavioral studies have made inferences about the nature of the computations by measuring the errors in the updating behavior during body translations. A gaze-independent mechanism could operate by storing and updating in Cartesian body-centered coordinates, which simply requires the amount of updating to be the same for each object: the opposite of the amount of body translation (Medendorp et al. 1999). If the translation is misjudged in this case, updating errors arise that have the same magnitude and the same sign irrespective of the depth of the target relative to the fixation. However, we recently falsified this model by showing that updating errors during active and passive body translations increase with depth from fixation and reverse in sign for objects presented at opposite depths from fixation (Clemens et al. 2012; Van Pelt and Medendorp 2007). Thus these gazecentered errors indicate that the internal remapping mechanism accounts for the geometry of motion parallax, although not perfectly. The neural correlate of such a parallax-sensitive updating mechanism, however, has not been revealed.

In the present study, we recorded electroencephalographic (EEG) signals while human subjects had to retain object locations during whole-body translation. We focused our analysis on spectral power in the alpha band $(8-12 \mathrm{~Hz})$, which not only is the dominant frequency band in the brain but also has been shown to remap across hemispheres when saccadic eye 
movements reverse the side of the remembered target relative to gaze (Van Der Werf et al. 2013).

Here, by exploiting the direction selectivity of alpha-band power, we tested whether internal representations of remembered visual objects are remapped transhemispherically when their representation reverses side relative to gaze because of passive whole-body translation dependent on their depth relative to the fixation point. We further investigated whether a relationship exists between the observed alpha power modulations during this remapping and the behaviorally observed errors.

\section{MATERIALS AND METHODS}

Participants. Sixteen healthy participants (9 women, 7 men; age range 18-31 yr), free of any known vestibular or neurological disorder and with normal or corrected-to-normal vision, gave their informed consent to participate in the experiment. All but one were right-handed. During analysis, data of 5 participants were discarded, resulting in 11 data sets (see Analysis-EEG for details).

The study was approved by the local ethics committee. Participants never received any feedback about their performance.

Setup. Subjects were seated in a custom-made linear sled designed to impose body motion along a lateral track of $800 \mathrm{~mm}$. The sled, powered by a linear motor (TB15N, Technotion, Almelo, The Netherlands), was controlled by a Kollmorgen S700 (Danaher, Washington, DC) drive. Motion kinematics of the sled were controlled with an accuracy better than $50 \mu \mathrm{m}, 2 \mathrm{~mm} / \mathrm{s}$, and $150 \mathrm{~mm} / \mathrm{s}^{2}$. The sled was configured such that participants were seated on the sled with their interaural axis aligned with the motion axis. Participants were restrained with a five-point seat belt. Head motion was restrained with a modified over-ear headphone fixed to the sled. Emergency buttons at both sides of the sled chair enabled subjects to stop the sled's motion immediately if needed. Psychophysical responses were recorded with a joystick, which was operated with the subject's right hand.

Positions of both eyes were continuously recorded at $500 \mathrm{~Hz}$ with an Eyelink II system (SR Research, Kanata, ON, Canada; accuracy < $0.5^{\circ}$ ). Its camera system, which was mounted to the sled, remained stable with respect to the head during the entire experiment.

The sled was further equipped with a 96-channel active electrode EEG system (Brain Products, Gilching, Germany). EEG data were recorded continuously during the experiment. The over-ear headphone prohibited EEG recordings from electrodes located around the ears (FT9/10, T7/8, TP7/8, and TP9/10), leaving 88 active recording sensors. Additionally, horizontal and vertical electrooculograms (EOGs) were recorded with electrodes placed below and above the right eye and at the bilateral outer canthi. Impedance of all electrodes was kept below $20 \mathrm{k} \Omega$, an adequate level for this active system. EEG and EOG signals were sampled at $1,000 \mathrm{~Hz}$ (amplifier bandwidth $0.016-1,000 \mathrm{~Hz}$, internal sampling rate $5 \mathrm{kHz}$ ) and then saved to disk.

Visual stimuli were presented with red light-emitting diodes (LEDs). A single LED served as an earth-stationary fixation point and was located 1,200 $\mathrm{mm}$ from the eyes when the sled was at the center of its movement range. Furthermore, a 450-mm-wide array of LEDs, consisting of 180 LEDs with a spatial separation of $2.5 \mathrm{~mm}$ (center to center), was used to present target and probe stimuli (see paradigm). The LEDs were approximately square $(2 \mathrm{~mm} \times 2 \mathrm{~mm})$, USB-powered with a luminance of 1 lumen or less. This array was oriented in parallel with the sled movement axis, at a distance of either $287 \mathrm{~mm}$ in front of fixation (913 $\mathrm{mm}$ from the eyes) or $535 \mathrm{~mm}$ behind fixation $(1,735 \mathrm{~mm}$ from the eyes), such that the retinal eccentricity was the same for targets behind and in front of fixation $\left(2.9^{\circ}\right)$. This way, the angle of rotation (and thus the "speed of movement" of the remembered target) was kept identical for targets behind and in front of fixation. The fixation point and LED array were displaced vertically by a few millimeters, such that they did not occlude each other and were approximately at eye level. The experiment was controlled with custom software programmed in a Delphi environment (CodeGear RAD Studio, Embarcadero Technologies, San Francisco, CA). EEG and eye movement data were recorded with separate computers, controlled by the main stimulus computer. Triggers were sent by the main stimulus computer to the EEG computer to synchronize task events and EEG data.

Task. The experiment took place in a completely darkened room, except for the stimulus lights. Subjects performed a forced-choice (left/right) spatial updating task, illustrated in Fig. 1. This paradigm is similar to that of Clemens et al. (2012), but with discrete sled motion (i.e., a single $400-\mathrm{mm}$ movement with a minimum jerk profile) to the left and right, as opposed to the continuous sinusoidal sled motion. A trial started with the sled $200 \mathrm{~mm}$ displaced from its center position, to either the right or left. The central fixation point, now $9.5^{\circ}$ away from the body midline, was turned on and had to be fixated until a probe appeared (0-3 s). Next, after $1 \mathrm{~s}$ the target was flashed $(50 \mathrm{~ms})$ either in front of or behind the fixation point. Both the fixation point and target were located on a line perpendicular to the sled motion axis at the center of the sled motion range. After a second delay ( $1 \mathrm{~s})$, the chair moved with a bell-shaped velocity profile (peak velocity 0.75 $\mathrm{m} / \mathrm{s}$, duration $1 \mathrm{~s}$ ) over a distance of $400 \mathrm{~mm}$ to the opposite position relative to center of the motion range. Next, after another delay (1 s) a probe stimulus was flashed for $50 \mathrm{~ms}$, after which fixation constraints were released. Using a left-right joystick response, subjects had to indicate whether the probe was located left or right in space relative to the remembered target. After the response, the next trial started with the current position of the sled as the new starting position.

The subject's task was thus to encode the initial target, update its memorized location over the course of the sled motion, and compare the internally updated location with the location of the probe. The retinal position of the target was jittered slightly $\left( \pm 0.6^{\circ}\right)$ across trials to deter stereotyped responses. Targets of the present trial were thus not informative about the location on the previous trial, avoiding possible feedback on the previous trial performance. Thirteen fixed target-probe differences were tested (equally spaced between $-4.6^{\circ}$ and $+4.6^{\circ}, 8$ repetitions for each difference), with a pseudorandomized target-probe difference in each trial. This fixed-interval psychometric procedure provided an estimate of the bias and precision of spatial updating across whole-body motion.

In total, there were four task conditions: two motion directions (left/right) and two depths of the target and probe ( $535 \mathrm{~mm}$ behind or $287 \mathrm{~mm}$ in front of fixation). Left and right trials alternated continuously, while target and probe depth alternated every two blocks.

The experiment aimed to capture activity related to updating of remembered spatial locations with intervening motion. To obtain a baseline for the EEG recordings that captures activity evoked by the translation itself, subjects were asked to keep fixation while their body was translated but no targets or probes were shown.

The experiment consisted of 11 blocks, 4 blocks of trials with targets/probes in front of fixation (the "front" condition), 4 blocks with targets/probes behind fixation (the "behind" condition), and 3 baseline blocks. Conditions alternated every two blocks. Baseline blocks followed after every two task blocks. A task block consisted of 52 trials (26 leftward and 26 rightward motion), while baseline blocks consisted of 70 trials (35 leftward and 35 rightward motion). As such, each of the four task conditions had 104 repetitions, while the baseline had 105 repetitions. The total experiment consisted of 626 trials, lasting $\sim 1 \mathrm{~h}$ (see Fig. $1 C$ ).

Analysis-behavior. All analyses were performed with MATLAB (MathWorks, Natick, MA). The forced-choice joystick responses were used to estimate the likelihood of a left response by fitting a 
Fig. 1. Schematic depiction of the setup and paradigm. $A$ : top view of the experimental setup. A typical trial started with the subject on either side of the central position. While the subject keeps fixation (depicted as a black dot) a target is flashed either in front of or behind the fixation point at $t=0 \mathrm{~s}$. Here, the target is presented behind fixation, thus to the right of gaze (see inset; star represents target). Subjects moved at $t=1 \mathrm{~s}$ with a bell-shaped velocity profile (distance $400 \mathrm{~mm}$, duration $1 \mathrm{~s}$ ) while keeping track of the (invisible) remembered target. Here, after the motion, the remembered target location (depicted as a dotted circle) is to the left of gaze. At $t=3 \mathrm{~s}$ a probe stimulus is flashed at the same depth, which could be left or right of the target. $B$ : time course of the experiment and position of the sled in an example trial. $C$ : example of condition order. Two blocks (52 trials) with fixation either behind (B) or in front of $(\mathrm{F})$ fixation were alternated, with a baseline block (Bl) in between. Start condition $(\mathrm{B} / \mathrm{F})$ was randomized across subjects.

A

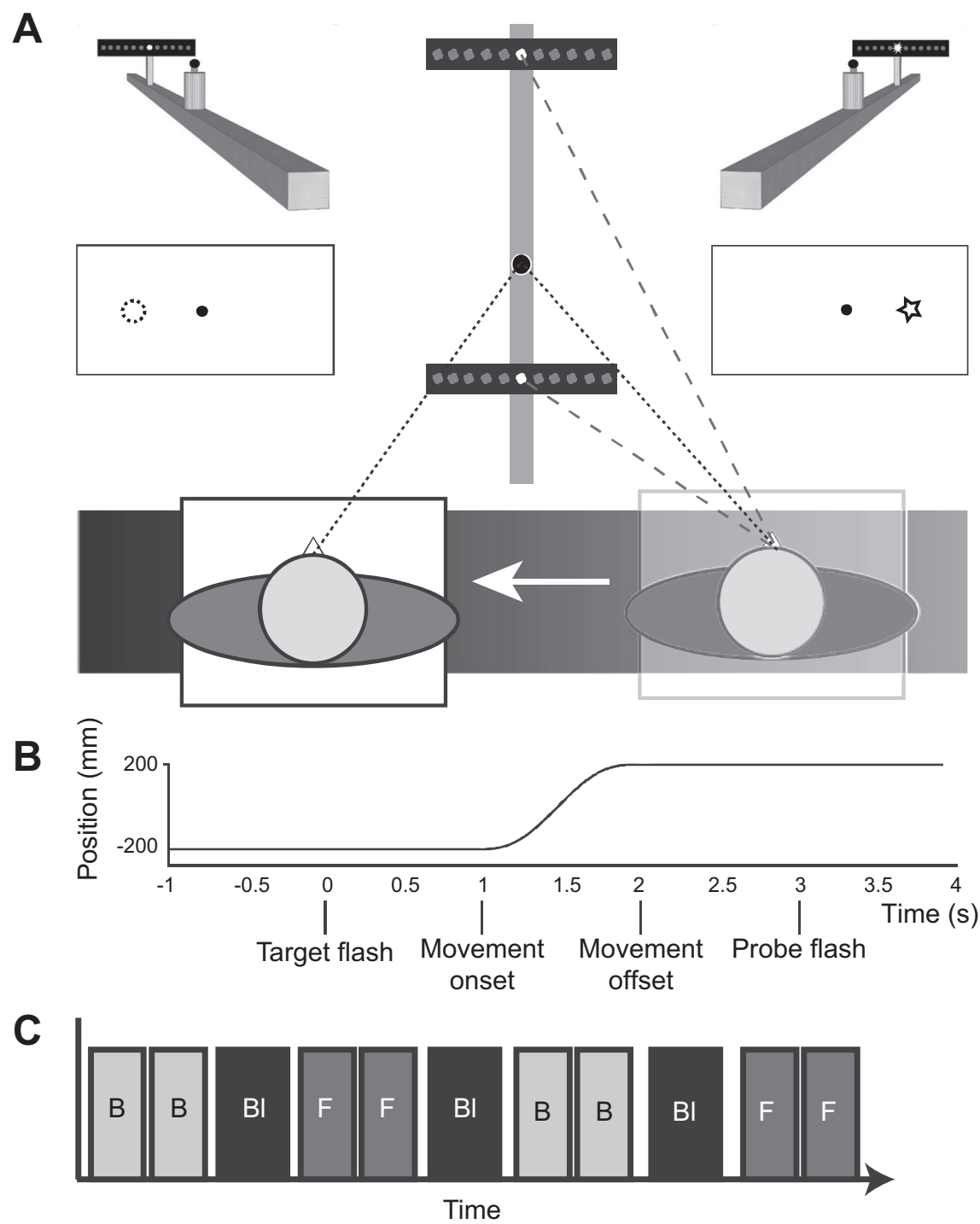

logistic function $f(x: \mu, \sigma)=\left(1+\exp \left(-\frac{2 \ln \left(\frac{1}{\alpha}-1\right)}{\sigma}(x-\mu)\right)\right)^{-1}$ with $\alpha=0.1$, using a Bayesian inference approach for each task condition using Psignifit 3.0 (Fründ et al. 2011). The mean of this function $(\mu)$ represents the bias (positive value corresponds to a leftward bias). The width of the curve $(\sigma)$, determined over the interval in which the function rises from $0.1(\alpha)$ to $0.9(1-\alpha)$, is inversely related to precision and serves as a measure of the participant's variability in the updating task.

To obtain a measure of updating performance independent of motion direction and target/probe depth, the observed biases were converted into an updating gain. Our previous study showed that biases can be consistently explained by a gaze-centered updating model with a nonunity gain factor between the actual $(T)$ and perceived $(\tilde{T})$ lateral translation (Clemens et al. 2012). In brief, let $O F$ be the vector from the cyclopean eye to the fixation point and $O R$ the vector from the cyclopean eye to the reference target. This allows us to express the true translation as the angle through which the eye rotates $\left[\sin \left(\phi_{F}\right)=T /|O F|\right]$ and that the reference target moves through $\left[\sin \left(\phi_{R}\right)=T /|O R|\right]$. Then, if we drop the sine because of small angles, to compensate for the translation, the target needs to be internally rotated by $\phi \cong \gamma \cdot T(1 /|O R|-1 /|O F|)$ relative to gaze. However, if the perceived translation has a nonunity gain $\gamma$, it follows that $\phi=\gamma \cdot \phi$, which results in angular bias $\phi-\phi$ (see Clemens et al. 2012 for further details). This angular bias can be expressed in Cartesian coordinates and thus $\mu$ of the psychometric function fits, by $\mu=(\phi$ $-\phi)|O R|=(\gamma-1) \cdot T(1-|O R| /|O F|)$. Provided that $O R, O F$, and $T$ are controlled parameters in our experiment and $\mu$ comes from our psychometric curves, we can calculate the translation gain. As can be noted, the bias flips sign according to presenting the target in front of $(O R<O F)$ or behind $(O R>O F)$ the fixation point, following the geometry of motion parallax.

Analysis-EEG. Analyses of EEG data were performed with the FieldTrip toolbox (Oostenveld et al. 2011) to obtain topographic maps of alpha activity and time-frequency representation of the lower frequency bands. All trials were manually checked for bad channels and/or artifacts in the time domain. While conditions were not blinded during artifact rejection, no electrode location (and thus laterality) could be derived from the raw traces. EOG recordings were used to detect blinks, loss of fixation, and saccades toward the targets or probes. Trials containing artifacts during the critical 3-s period ranging from target onset until probe presentation (e.g., blinks, saccades, muscle twitches, signal loss, jumps) were removed, and bad channels were interpolated with a distance-weighted nearest-neighbor approach. Eye tracking data were used to check for residual eye movements in the cleaned data. Two subjects showed excessive blinks and eye movements, leading to a trial rejection above $50 \%$, and were 
excluded from further analysis. Because of the importance of keeping fixation in this task we chose to delete these trials, rather than using a correction method. In three subjects, the behavioral data could not be fitted because of poor performance in one or more conditions. This was mainly because the biases $(\mu)$ of these subjects were beyond the range of sampled probe locations. Because our analyses require a coupling between the EEG data and behavior, these subjects were excluded from further analysis. This resulted in 11 data sets. Of the remaining subjects, mean trial rejection rate was $20.5 \%$ (SD 9.0) for task trials and $25.9 \%$ (SD 8.2) for baseline trials. Rejection rates of the task trials did not significantly differ between left and right direction or target depth (all $P>0.29$ ) For the remaining trials, EEG signals were low-pass filtered off-line at $80 \mathrm{~Hz}$ and high-pass filtered at $1 \mathrm{~Hz}$. A two-pass band-stop filter $(48-52 \mathrm{~Hz})$ was applied to reduce line noise. Before frequency analysis, the data were rereferenced to "average reference" (Bertrand et al. 1985).

The full power spectrum was calculated for each 4-s trial, aligned to the presentation of the target (or the equivalent time point in the baseline trials) with Morlet wavelets ( $\sigma=7$, resulting in an average frequency resolution of $2.8 \mathrm{~Hz}$ and a $222-\mathrm{ms}$ wavelet length in the alpha range), over a frequency range of $1-80 \mathrm{~Hz}$. Data were averaged and $z$-transformed. For each condition, the corresponding baseline condition was subtracted, effectively subtracting out task-irrelevant activity, such as low-level vestibular processing (e.g., baseline condition moving left was subtracted from the task conditions containing leftward motion). Data were further analyzed in two different ways: first, to show the average task-evoked activity and second, to isolate the lateralized components.

The analyses focused on power modulations in the alpha band $(\sim 8-12 \mathrm{~Hz})$. For each subject, average power spectra were calculated to locate the peak alpha frequency. The mean alpha peak was centered at $10.3 \mathrm{~Hz}$ (SD 0.46).

To distinguish the task-evoked activity, baseline-corrected data were pooled by mirroring conditions with opposite visual input and averaged over conditions. Thus, in this analysis, data of the front-right (FR) condition (motion to the right with targets presented in front of fixation) and the behind-left (BL) condition (leftward motion, target behind fixation), which both have the initial target in the right visual hemifield, were mirrored according to the anterior-posterior midline (left and right hemispheres were swapped) and summed with the front-left (FL) and behind-right (BR) conditions. Thus the taskevoked component was computed as (FL $\left.+\mathrm{FR}^{*}+\mathrm{BL}^{*}+\mathrm{BR}\right) / 4$, in which $*$ indicates a mirror flip of the data. Here, the right hemisphere is contralateral to initial target presentation.

To isolate the lateralized components, conditions with opposite initial visual input were subtracted, i.e., data of the FR condition were subtracted from the FL condition, as were the data of the BR condition from the BL condition data. These two data sets were then subtracted again. Thus the lateralized component was calculated as (FL - FR) $(\mathrm{BL}-\mathrm{BR})$. Here also, the right hemisphere is contralateral to the initial target presentation.

To assess whether the observed power modulations in the alpha band are due to spatial updating performance, we correlated the power difference before and after the motion with the individual subjects' behavioral gains. To this end, a function was fitted describing the time course of alpha power per electrode, for all conditions. The function assumes a constant alpha power over the premotion period, a linear change in alpha power after motion onset, and again a constant alpha power in the postmotion baseline period. The pre- and postbaseline amplitude, change onset, and postbaseline onset were free parameters. The difference in alpha power before and after motion, reflecting the alpha modulation during the trial, was used in an independent-samples regression analysis against the gain factor. Statistical significance testing was done by using cluster-based statistics and Monte Carlo permutation tests to correct for multiple comparisons (Maris and Oostenveld 2007).

\section{RESULTS}

We tested whether the neural mechanism for visual stability takes the geometry of motion parallax into account when updating target locations during translational body motion. Subjects had to remember the location of a target, briefly flashed in front of or behind the eyes' fixation point. After an intervening whole-body translation, they had to report the memory of this location using a left/right choice response (i.e., whether a probe was perceived left or right of the memorized target). We first summarize the behavioral results, followed by an analysis of spectral power modulations in the alpha band and the link between these two.

Behavior. Figure $2 A$ shows the psychometric data of a typical subject, separately for the front and behind conditions (Fig. 2A, top vs. bottom). The respective panels plot the fraction of leftward responses (indicated by circles) as a function of horizontal probe location relative to the initial target, together with the fitted psychometric function. If updating performance were perfect, the psychometric curves would be centered at zero. However, the actual results show consistent biases, which flip between front and behind conditions, as well as for leftward versus rightward motion. A body-centered updating model would imply that updating errors should have the same sign for targets in front of and behind the fixation point, depending only on the position of the target relative to the body. However, the depth-dependent error reversals that are seen indicate that targets are coded and updated relative to gaze. In other words, these observations suggest that the updating mechanism simulates the geometry of motion parallax, by coding and updating targets relative to gaze.

We derived estimates of the bias $(\mu)$ values in each of the four conditions. Figure $2 B$ depicts these values for all subjects (dots), in top-view panels, separately for leftward and rightward motion. Bias values range approximately between -13 and $13 \mathrm{~cm}$ and generally reverse for targets presented in front of versus behind fixation. Bias values were entered in a repeated-measures ANOVA with motion direction (leftward/ rightward) and target location (front/behind) as factors. While there were no significant main effects (all $P>0.21$ ), it revealed a significant Motion Direction $\times$ Target Location interaction $\left[F(1,10)=7.15, P=0.023\right.$, partial $\left.\eta^{2}=0.42\right]$. Post hoc analyses showed that conditions that differ in either motion direction or target location, but not both, are significantly different (all $P=<0.036$ ) while conditions that differ in both are not (all $P>0.14$ ). This supports the notion that updating is performed in a gaze-centered reference frame, consistent with the findings of Clemens et al. (2012).

To determine the quality of updating, we applied the gazecentered model described in MATERIALS AND METHODS to calculate the gain value for each individual subject. Figure $2 C$ depicts the resulting gain values, which range from 0.2 to 1.4 across subjects. In other words, the subject group is not homogeneous with respect to gain; there are good (gain near 1) and less good (median split by gain 0.71 ) performers. Furthermore, the gain did not significantly differ among the four conditions (repeated-measures ANOVA, $P=0.15$ ), which means that the model also correctly accounts for the sign of the updating bias, reversing dependent on target depth. Thus the geometry can explain the bias across all four conditions, suggestive of a gaze-centered parallax-sensitive mechanism 
Fig. 2. Behavioral results. A: psychometric curve fits for a single subject for the leftward and rightward motion conditions with the targets presented in front of fixation (top) or behind fixation (bottom). Bias $(\mu)$ values (dotted vertical lines), corresponding to the 0.5 probability $(P)$ point, were extracted for every subject. To construct the curve, all task trials were included (104 per condition). B: bias $(\mu)$ of the psychometric curve fit for the 2 target/probe depths and left and right motion directions for all subjects. The data point marked with a dark circle corresponds to the single-subject data in $A$. Fixation depth is shown as a dotted horizontal line. $C$ : gain factors $(\gamma)$ for the 4 task conditions.
A

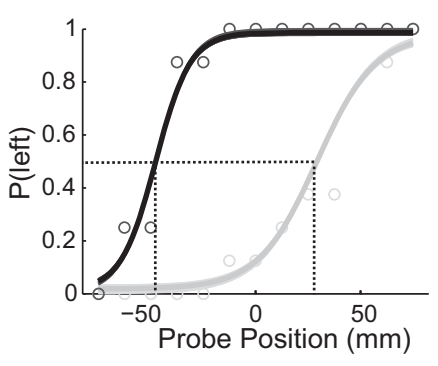

Rightward motion Leftward motion

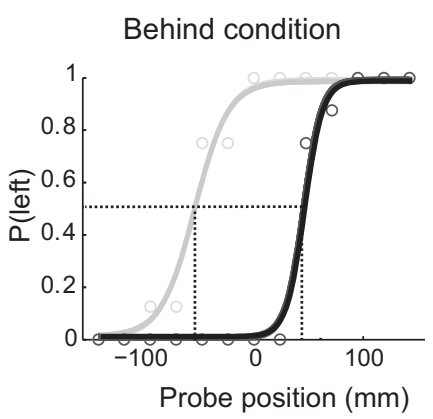

B
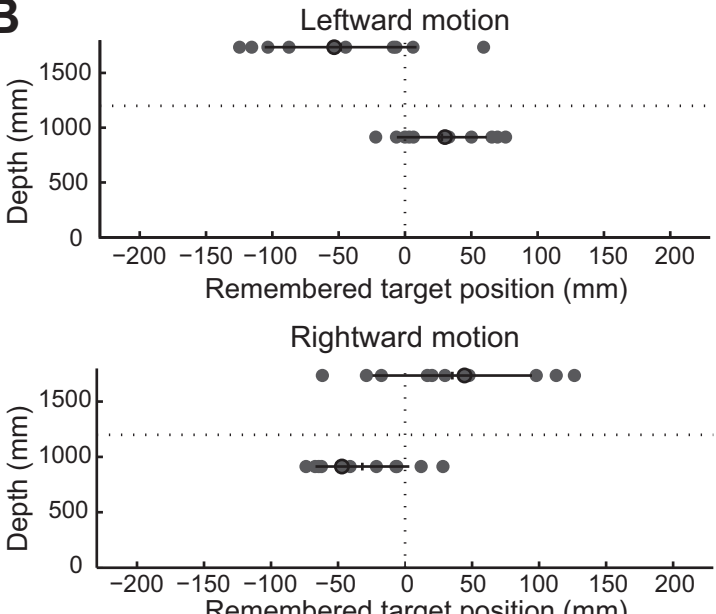

C

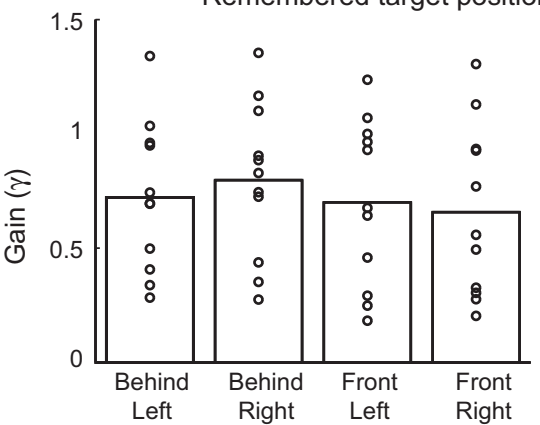

underlying visual stability. The question is: Can these results be linked to a neural mechanism that reflects these principles?

Alpha-band power simulates motion parallax. The behavioral observations above, as well as previous physiological work on head-fixed saccade and smooth pursuit remapping, predict that certain brain areas should have an activity pattern that evolves during body motion in a way that depends on object depth. In the present paradigm, remembered targets at opposite depths from fixation reverse (left-right) with respect to gaze direction during the body motion. We exploited the direction selectivity of spectral power to test whether these physical shifts are accompanied by a change in focus of alpha-band power from one hemisphere to the other to internally simulate this shift.

Figure 3 shows the group average scalp topography of the power modulations of the alpha band in the four task conditions relative to baseline, averaged across subjects, during four nonoverlapping time intervals, each covering a 0.5 -s interval. Power reductions are coded in cooler colors and power enhancements in warmer colors. Figure $3 A$ plots power during the leftward motion trials, with the target presented at $t=0 \mathrm{~s}$ in front of fixation, in the left visual field ("front left" condition). This stimulus response, observed in the subsequent, premotion time interval $(0-0.5 \mathrm{~s})$, is reflected by a clear power reduction at the occipito-parietal electrodes with an inclination to the right, contralateral hemisphere. During the acceleration phase of the motion, from 1 to $1.5 \mathrm{~s}$, the reduction diminishes and shifts focus to the left hemisphere during the deceleration phase of the motion (1.5-2 s), which is the hemisphere contralateral to updated target location relative to gaze. The left hemisphere power reduction is maintained during the postmotion delay (2.5-3 s) until the probe appears (at $3 \mathrm{~s}$ ).
Using the same format, Fig. $3 B$ plots the results of the leftward motion trials with the target presented behind the fixation point, in the right hemifield ("behind left" condition). Compared with Fig. $3 A$, the pattern is now reversed; although weaker, the initial stimulus-related power reduction of the left hemisphere is remapped to the right hemisphere in response to the intervening body translation.

Figure 3,C and $D$, show the power modulations during rightward motion trials, in the same format as Fig. 3, $A$ and $B$ ("front right" and "behind right" conditions). Again, both Fig. 3, $C$ and $D$, show an initial power reduction due to the initial stimulus. After the intervening motion, this suppression is reorganized from the hemisphere contralateral to the initial target location to the hemisphere ipsilateral to the initial target location for the front right condition (Fig. 3C). This pattern is less prominent for the behind right condition; while the initial power reduction of the contralateral hemisphere still vanishes during the motion, no clear reduction of the ipsilateral hemisphere has emerged (Fig. 3D). The relatively weaker lateralization in the behind condition may be due to larger variability in remembered target position, which can be seen in the behavior as well (Fig. 1B). Because all four panels of Fig. 3 show data relative to baseline (i.e., the same motion without updating targets), their general result can be interpreted as indicative of a gaze-centered transhemispheric remapping of target representations during translational motion.

To show the consistency of these patterns, Fig. $4 A$ shows the scalp topography of the common task-evoked, subject-specific alpha component that is shared across all four conditions relative to the baseline condition. Note that, in plotting this contrast, the convention is that left and right hemispheres are ipsilateral and contralateral to the gaze-centered location of the 

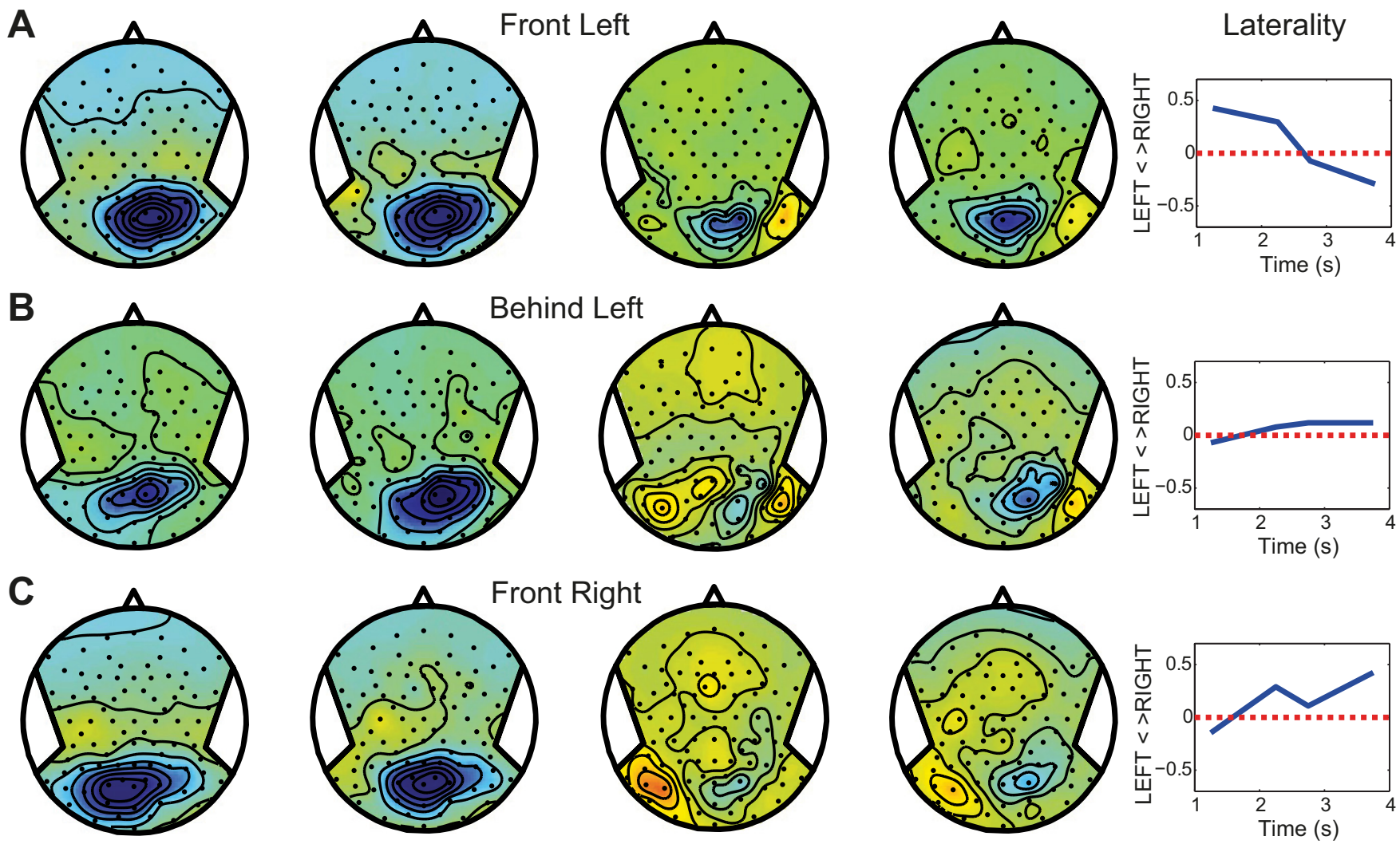

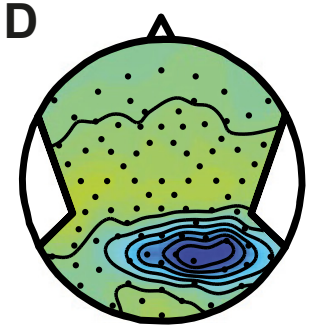

0-0.5s

pre-motion

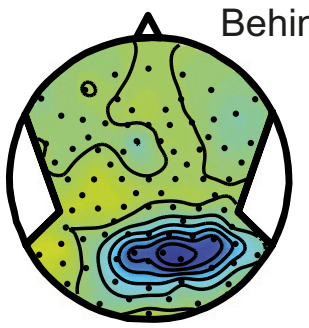

1-1.5s acceleration

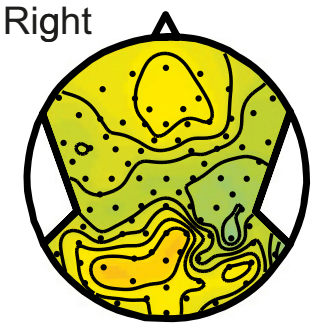

1.5-2s deceleration

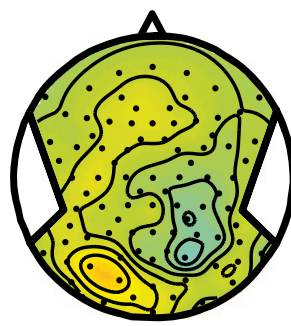

2.5-3s post-motion

Fig. 3. Group average scalp topography of alpha-band power at 4 different time intervals, separately for the 4 conditions of the experiment: front left $(A)$, behind left $(B)$, front right $(C)$, behind right $(D)$. The 1st time interval $(0-0.5 \mathrm{~s})$ shows the oscillatory response to the visual presentation of the target. At $t=1 \mathrm{~s}$ motion commences (lasting $1 \mathrm{~s}$ ), and thus the 2 nd and 3rd time interval reflect the $1 \mathrm{st}$ and 2 nd half of motion, respectively. The 4 th time interval (2.5-3 s) reflects activity just before the presentation of the probe stimulus, when the subject is in a stationary situation again. Right: lateralization index over time, calculated as the normalized difference in alpha reduction for the left and right hemispheres. Positive laterality index indicates a right hemisphere bias and negative laterality index a left hemisphere laterality bias.

initial stimulus, respectively. Regions with warmer (red) colors indicate a relative power increase; regions with cooler (blue) colors represent a relative power decrease. The scalp topography is shown at the same time intervals as in Fig. 3. The initial, stimulus-driven, response observed in the premotion time interval $0-0.5 \mathrm{~s}$ is reflected by a clear decrease in power in the contralateral hemisphere, extending to the central parietal electrodes. During motion, the power reduction becomes weaker and shifts focus to the left hemisphere, which is contralateral to the gaze-centered location of the updated stimulus location. To test this further, a parieto-occipital region of interest was defined for each hemisphere (P1/2, P3/4, P5/6, P7/8, PO3/4, $\mathrm{PO} 7 / 8$, PO9/10, PPO9/10, O1/2, and O9/10), the mean activities of which were extracted for a premotion time window $(0-0.5 \mathrm{~s})$ and a postmotion time window (2.5-3 s). These values were entered in an ANOVA with factors Time (pre/ post) and Hemisphere (ipsilateral/contralateral with respect to initial target). This revealed a significant Time $\times$ Hemisphere interaction effect $\left[F(1,10)=15.98, P=0.003\right.$, partial $\eta^{2}=$ $0.62]$, supporting the idea that the target representation is actually remapped.

To further analyze the laterality of these activity patterns, the lateralized components were isolated as the power differences between the two hemispheres. Figure $4 B$ shows the scalp topography of the lateralized components. Here also, an initial reduction can be seen in the right, contralateral hemisphere. Subsequently, tracking the topography across the various time intervals indicates a polarity reversal during the translation, i.e., when targets must be remapped to the ipsilateral side of gaze. To test the significance of the remapping effect, we 
A

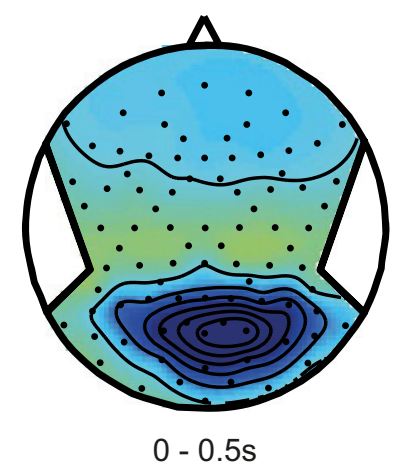

B

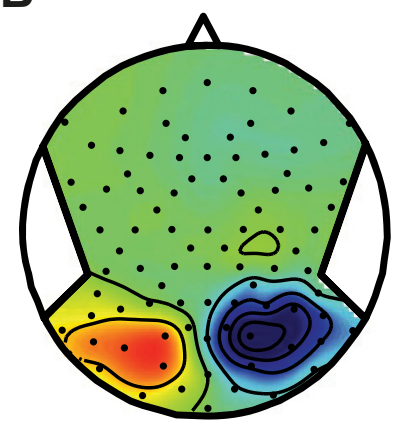

Task-evoked activity

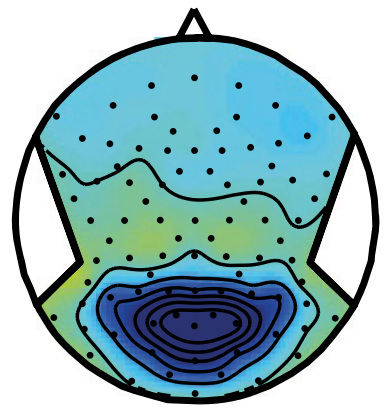

$1-1.5 s$

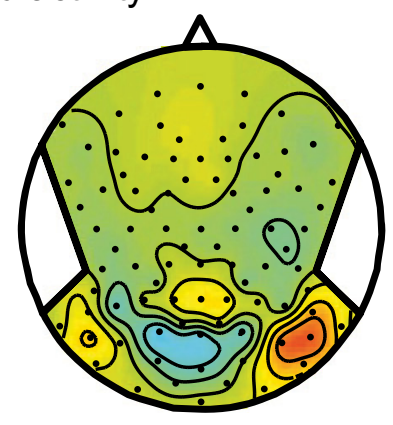

$1.5-2 \mathrm{~s}$

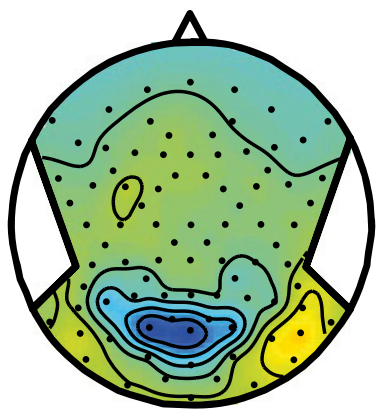

$2.5-3 s$
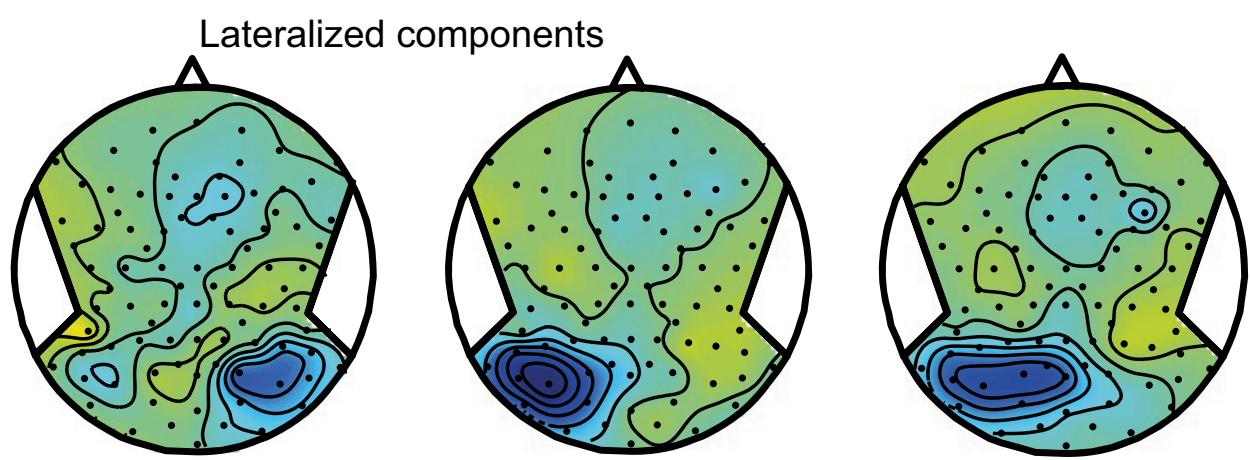

Pre-post modulation
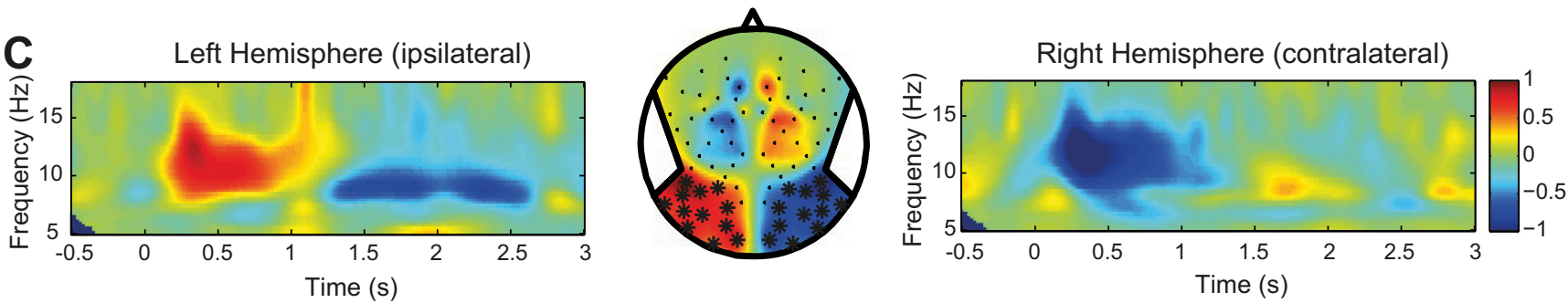

Fig. 4. Isolating the group average task-evoked activity and the lateralized components of the alpha-band activity across all conditions. A: task-evoked activity during the 4 time intervals of Fig. 3. Warmer colors, increase in alpha power; cooler colors, decreases in power. Plotting convention: the right hemisphere is contralateral to the initially presented target, and the left hemisphere is contralateral to the updated target position relative to gaze. $B$ : lateralized components, illustrated by a subtraction of all conditions. Plotting conventions as $A$. $C$, center: electrodes that show a significant $(* P<0.002)$ difference between alpha power before $(0-0.5 \mathrm{~s})$ and after $(2.5-3 \mathrm{~s})$ the motion in the subtraction. Left and right: time-frequency plots of the lower-frequency band of the lateralized components for these significant electrodes (TPPP7/8h, P3/4, P5/6, P7/8, P9/10, PO3/4, PO7/8, PO9/10, PPO9/10h, O9/10, OI1/2h, O1/2).

compared the difference in power before and after the motion (time windows $0-0.5 \mathrm{~s}$ and $2.5-3 \mathrm{~s}$ ) using a dependentsamples $t$-test with cluster permutation correction. As shown in Fig. $4 C$, center, this revealed a significant bilateral (mirror symmetric) cluster, as marked by the asterisks $(P<0.002)$. To demonstrate the temporal dynamics of the remapping effect in more detail, Fig. 4, C, left and right, depict the time-frequency plots of the lower frequency band of the lateralized component contrast. Again, the contralateral hemisphere (Fig. 4C, right) shows initial power reduction in the poststimulus phase before motion onset. This reduction is remapped to the ipsilateral hemisphere (Fig. 4C, left) during motion $(t=1-2 \mathrm{~s})$ and is sustained after motion ends.

Alpha-band modulations correlate with updating gain. If the observed power modulations in the alpha band are indeed part of a gaze-centered updating mechanism, they should vary in relation to updating performance. The behavioral performance of our subjects varied between low and high gain (see Fig. 2). Figures 3 and 4 show that the updating process is reflected in a difference in alpha-band activity before and after translation. To quantify this, the modulation in the alpha band over time was calculated for all electrodes per subject and averaged according to hemisphere (contra- or ipsilateral). This pre-post difference value was entered in a regression analysis with the gain factor. This yielded a significant cluster of electrodes $(P<$ 0.025 cluster corrected), as shown in Fig. $5 A$. This bilateral parietal cluster shows a significant negative correlation with updating performance. This means that a larger difference between the alpha power before and after motion is associated with a higher gain factor, i.e., better performance. This is illustrated in Fig. 5B, where the alpha modulation data have been median-split into a high- and a low-performance group. As can be seen, "good" performers (gain > 0.71) show larger alpha modulations at more anterior, central parietal electrodes, 


\section{A Behavioral correlation}

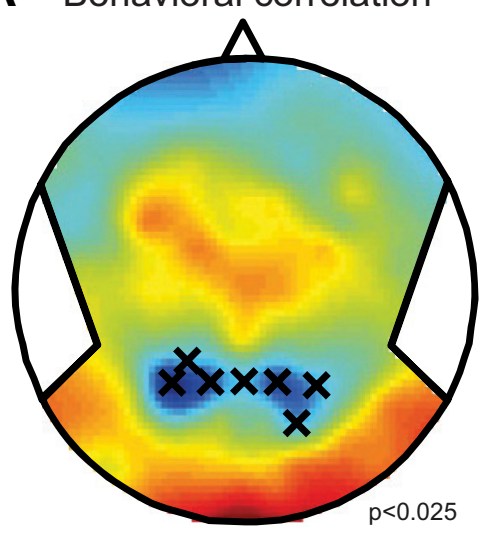

B High Gain subjects

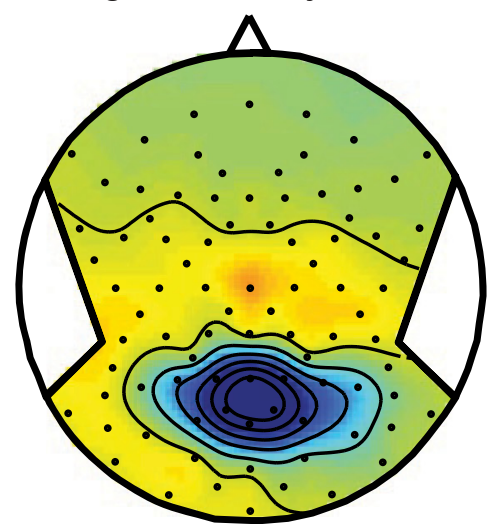

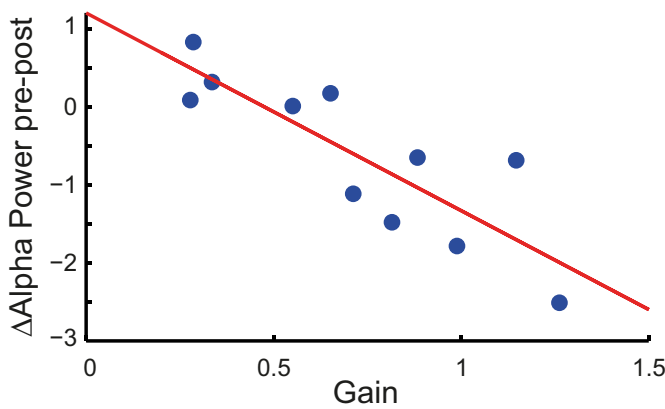

Low Gain subjects

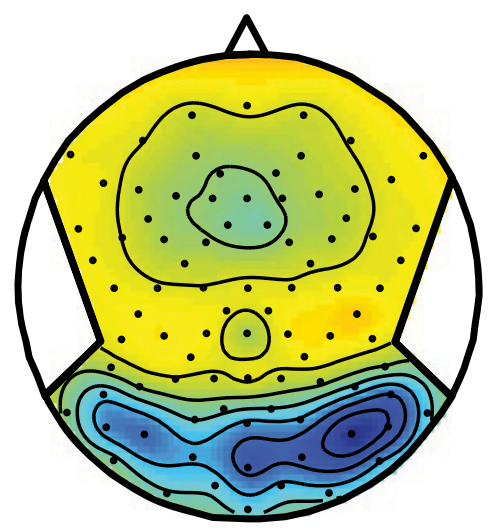

Fig. 5. Linking behavioral and neural correlates. $A$ : electrodes showing a significant correlation between power modulations in the alpha band and psychometrically determined updating gain. Color map: $t$ values from the regression analysis. Right: relationship between alpha power and updating performance at significant sensors $(\mathrm{Pz}, \mathrm{P} 1$, P3, P4, CCP3h, and PO4). B: scalp topography plot of the alpha modulation (pre- and postmotion difference) over time as used in the regression analysis with gain for "good" (left) and "bad" (right) performers (median split, gain $>0.71$ considered "good"). Cooler colors indicate a greater pre- - postmotion difference and thus a greater modulation of alpha over time. whereas "poor" performers show more posterior modulations at occipital electrodes. This shows that reductions in the alpha band at parietal electrodes during task performance are clearly associated with accurate spatial updating.

\section{DISCUSSION}

Using saccadic updating paradigms, it has been suggested that the brain stores dynamic visual representations within a gaze-centered reference frame to maintain visual stability (Bellebaum and Daum 2006; Medendorp et al. 2003a; Merriam et al. 2003; Van Der Werf et al. 2013). Here we studied whether similar gaze-centered mechanisms are recruited for visual stability across whole-body motion. For gaze-centered updating mechanisms to be veridical under whole-body motion during world-stationary fixation, parallax geometry needs to be taken into account. Importantly, our subjects could not see the effects of parallax geometry but had to simulate it internally based on vestibular motion signals and other extraretinal motion cues, combined with estimates of fixation and target depth. Our focus was on the role of alpha-band oscillations, which have been implicated in saccadic updating (Van Der Werf et al. 2013). By exploiting the direction selectivity of alpha-band power, we show parallax-sensitive, transhemispheric remapping of alpha-band power when remembered targets cross hemifields relative to the fixation point, dependent on their depth. At parietal electrodes, the strength of these remapping modulations correlated significantly with the quality of the visuospatial updating, pointing to a crucial role of this region in maintaining visual stability.

Our behavioral results show that subjects are able to perform (although not perfectly) the necessary update of spatial location for an intervening passive whole-body motion, which is in line with previous studies (Li et al. 2005; Medendorp et al. 2003b; Van Pelt and Medendorp 2007). The direction of the systematic errors, or biases (a deviation to the left or right of the veridical location), depended on the depth of the target and motion direction of the subject, consistent with the findings of Clemens et al. (2012). Thus from the behavior we infer that the brain employs a gaze-centered mechanism to internally update remembered visual space during whole-body translations, taking the geometry of motion parallax into account.

The behavioral data could be explained by a gaze-centered updating model with a gain factor on the translation. Gain factors were below 1 (mean $\sim 0.7$ ), indicating a general underestimation of translation, which is in line with a general underestimation of distance traveled (Tremblay et al. 2013). However, performance was better than previously found with passive sinusoidal translations (Clemens et al. 2012), suggesting that the content of vestibular feedback (sinusoidal vs. transient) and task conditions affect updating performance. Other studies, using active motion (i.e., where additional information is available in the form of a motor command copy), show a close to ideal performance (Medendorp et al. 2003b; Van Pelt and Medendorp 2007), suggesting that visual stability 
essentially requires a multimodal solution (Klier and Angelaki 2008; Medendorp 2011).

The incorporation of the geometry of motion parallax was not only observed in the behavioral biases. Here, for the first time, we show a neural correlate in terms of time-varying alpha-band activity over occipito-parietal areas. By choosing a baseline that included the translation, but no updating of spatial targets, activity was isolated that reflected spatial updating. In all four of our updating conditions, presentation of the initial target is followed by a contralateral decrease in alpha power over occipital and occipito-parietal electrodes in the premotion phase. This initial power decrease is driven by the visual input of the target. Importantly, after the intervening motion, alpha power reduction is sustained in the opposite hemisphere during the postmotion phase, contralateral to the updated location of the reference target in gaze coordinates, in the absence of direct visual stimulation of this hemisphere. Our results show that the location of the target is reflected in lateralized alpha reductions that remap transhemispherically during the updating task. This reversal in lateralization is similar to saccadic updating, where a transhemispheric remapping of power reductions from the hemisphere contralateral to the initial target before the saccade to the hemisphere contralateral to the updated target with respect to gaze after the saccade has been shown (Van Der Werf et al. 2013). However, in the present experiment an internal simulation of motion parallax is needed to work out the location of the updated target in gaze coordinates.

In the present paradigm, a spatial target had to be stored in memory and updated throughout the trial. Low-frequency oscillations, such as the alpha band $(8-12 \mathrm{~Hz})$ and the theta band $(5-8 \mathrm{~Hz})$, have been implicated in working memory processes (Jensen et al. 2002; Raghavachari et al. 2006; Sauseng et al. 2009). Whereas theta band oscillations seem to be involved in sequential processing (Hsieh et al. 2011; Roberts et al. 2013; VanRullen 2013), the alpha band seems to be more specific for (visuo)spatial memory tasks (Fries et al. 2001; Roux and Uhlhaas 2014; Sauseng et al. 2005), which is consistent with the present observations.

Various studies have further shown a negative coupling between power in the alpha band and power in the gamma band (Fries et al. 2001; Osipova et al. 2008). This frequency coupling is suggested to establish a coherent neuronal group representing a location in working memory (Salazar et al. 2012). Unfortunately, we were unable to obtain reliable measurements in the gamma band. Although the head was fixed relative to the body, the acceleration of the chair caused increased tension in the neck muscles, creating artifacts in the higher frequencies. In future work, it would be interesting to see if gamma is indeed phase locked to alpha during spatial updating performance and whether it is similar to saccadic updating (Van Der Werf et al. 2013).

Could the present results be explained as a purely attentional phenomenon? In the literature, alpha-band power has been strongly linked to visuospatial attention (Foxe et al. 1998; Worden et al. 2000; see for reviews Foxe and Snyder 2011; Jensen et al. 2012). Attending a visual hemifield results in lateralized increases and decreases in alpha power in the ipsiand contralateral hemispheres, respectively. Indeed, while the initial reduction is likely a response to the visual stimulation, the ensuing alpha reductions may be indicative of covert attention. Following this notion, the power modulations in the alpha band seen over the occipito-parietal areas may be an implementation of an "attentional pointer" to the remembered location in gaze coordinates (Cavanagh et al. 2010) and instantiate one of the mechanisms that contributes to the updating process. However, the update of this attentional pointer still requires an internal simulation of motion parallax, which is accompanied by time-varying spatial-selective alpha power reductions in the posterior brain.

We found that alpha-band activity at parietal electrodes correlated significantly with spatial updating performance for whole-body motion. Even though no source localization was performed and thus no anatomical structures can be implicated, we suggest that the resulting pattern of activity may have arisen from the neuronal computations that have to take place in parietal cortex. In particular, this may stem from the lateral intraparietal area (LIP), which is known to contain neurons with gaze-centered receptive fields that predictively remap the neural image to anticipate the visual consequences of saccades (Duhamel et al. 1992; Kusunoki and Goldberg 2003). Similar observations have actually also been made for the frontal eye fields (Sommer and Wurtz 2006). It is further known that the receptive fields of LIP neurons are three-dimensional (3D), meaning that they are tuned to a combination of target direction and target depth (Genovesio and Ferraina 2004; Gnadt and Mays 1995). It is well possible that such neurons with 3D receptive fields play a role in parallax-sensitive updating provided they also integrate vestibular or other extraretinal information about the motion. Indeed, there are pathways that transmit vestibular signals to parietal cortex (Kaufman and Rosenquist 1985; Meng et al. 2007; Shinder and Taube 2010), supporting this proposal. Also, human studies have explicitly implicated parietal cortex in self-motion processing based on caloric vestibular stimulation (Dieterich et al. 2003; Suzuki et al. 2001; see for review Lopez et al. 2012) and optic flow signals (Kovács et al. 2008; Wolbers et al. 2008). It seems plausible that the intersubject differences in alpha modulation in parietal cortex reflect the neural basis for more veridical integration of vestibular signals into the updating process, as the larger modulation is associated with better updating performance.

As pointed out by Van Pelt and Medendorp (2007), vestibular and retinal signals are not the only relevant signals to implement spatial updating in the present study. Also, changes in eye position to keep the eyes at the fixation point during the translation-the version and vergence eye movements-provide cues for the updating mechanism. These signals have also been observed in parietal cortex (Andersen et al. 1985; Chang and Snyder 2010; Gnadt and Beyer 1998; Gnadt and Mays 1995; Prevosto et al. 2009), where they need to be integrated with vestibular signals, as well as target depth and direction information, in order to compute the evolving representation of remembered $3 \mathrm{D}$ space during self-motion that we have shown.

Taken together, our results suggest that during passive motion the representation of a remembered and updated target is reflected in lateralized alpha-band activity. The pattern of activity in the alpha band is in line with a gaze-centered updating mechanism, necessitating inclusion of motion parallax effects. Activity at the posterior parietal electrodes shows a strong relation to updating performance, possibly indicating a crucial role for the posterior parietal cortex in updating locations in the world under passive motion conditions. 


\section{GRANTS}

This work is supported by grants from the European Research Council (EU-ERC 283567), an EU-FP7-FET grant (SpaceCog 600785), and the Netherlands Organisation for Scientific Research (NWO-VICI: 453-11-001; NWOVENI: 451-10-017).

\section{DISCLOSURES}

No conflicts of interest, financial or otherwise, are declared by the author(s).

\section{AUTHOR CONTRIBUTIONS}

Author contributions: T.P.G., L.P.J.S., and W.P.M. conception and design of research; T.P.G. performed experiments; T.P.G. analyzed data; T.P.G., L.P.J.S., and W.P.M. interpreted results of experiments; T.P.G. prepared figures; T.P.G. drafted manuscript; T.P.G., L.P.J.S., and W.P.M. edited and revised manuscript; T.P.G., L.P.J.S., and W.P.M. approved final version of manuscript.

\section{REFERENCES}

Andersen RA, Essick GK, Siegel RM. Encoding of spatial location by posterior parietal neurons. Science 230: 456-458, 1985.

Angelaki DE, Hess BJ. Self-motion-induced eye movements: effects on visual acuity and navigation. Nat Rev Neurosci 6: 966-976, 2005.

Bellebaum C, Daum I. Time course of cross-hemispheric spatial updating in the human parietal cortex. Behav Brain Res 169: 150-161, 2006.

Bertrand O, Perrin F, Pernier J. A theoretical justification of the average reference in topographic evoked potential studies. Electroencephalogr Clin Neurophysiol 62: 462-464, 1985.

Blohm G, Missal M, Lefèvre P. Interaction between smooth anticipation and saccades during ocular orientation in darkness. J Neurophysiol 89: 14231433, 2003.

Blohm G, Missal M, Lefèvre P. Processing of retinal and extraretinal signals for memory-guided saccades during smooth pursuit. J Neurophysiol 93: 1510-1522, 2005.

Bridgeman B, Nardello C. Conflict between aftereffects of retinal sweep and looming motion. Psychol Res 56: 78-82, 1994.

Cavanagh P, Hunt AR, Afraz A, Rolfs M. Visual stability based on remapping of attention pointers. Trends Cogn Sci 14: 147-153, 2010.

Chang SW, Snyder LH. Idiosyncratic and systematic aspects of spatial representations in the macaque parietal cortex. Proc Natl Acad Sci USA 107: 7951-7956, 2010.

Clemens IA, Selen LP, Koppen M, Medendorp WP. Visual stability across combined eye and body motion. J Vis 12: 8, 2012.

Colby CL, Goldberg ME. Space and attention in parietal cortex. Annu Rev Neurosci 22: 319-349, 1999.

Dash S, Catz N, Dicke PW, Thier P. Encoding of smooth-pursuit eye movement initiation by a population of vermal Purkinje cells. Cereb Cortex 22: 877-891, 2012.

Dieterich M, Bense S, Lutz S, Drzezga A, Stephan T, Bartenstein P, Brandt T. Dominance for vestibular cortical function in the non-dominant hemisphere. Cereb Cortex 13: 994-1007, 2003.

Duhamel JR, Colby CL, Goldberg ME. The updating of the representation of visual space in parietal cortex by intended eye movements. Science 255: 90-92, 1992.

Foxe JJ, Simpson GV, Ahlfors SP. Parieto-occipital approximately $10 \mathrm{~Hz}$ activity reflects anticipatory state of visual attention mechanisms. Neuroreport 9: 3929-3933, 1998.

Foxe JJ, Snyder AC. The role of alpha-band brain oscillations as a sensory suppression mechanism during selective attention. Front Psychol 2: 154, 2011.

Fries P, Reynolds JH, Rorie AE, Desimone R. Modulation of oscillatory neuronal synchronization by selective visual attention. Science 291: 15601563, 2001.

Fründ I, Haenel NV, Wichmann FA. Inference for psychometric functions in the presence of nonstationary behavior. J Vis 11: 16, 2011.

Genovesio A, Ferraina S. Integration of retinal disparity and fixation-distance related signals toward an egocentric coding of distance in the posterior parietal cortex of primates. J Neurophysiol 91: 2670-2784, 2004.

Gnadt JW, Beyer J. Eye movements in depth: what does the monkey's parietal cortex tell the superior colliculus? Neuroreport 9: 233-238, 1998.
Gnadt JW, Mays LE. Neurons in monkey parietal area LIP are tuned for eye-movement parameters in three-dimensional space. J Neurophysiol 73: 280-297, 1995.

Hsieh LT, Ekstrom AD, Ranganath C. Neural oscillations associated with item and temporal order maintenance in working memory. J Neurosci 31: 10803-10810, 2011.

Jensen O, Bonnefond M, VanRullen R. An oscillatory mechanism for prioritizing salient unattended stimuli. Trends Cogn Sci 16: 200-206, 2012.

Jensen O, Gelfand J, Kounios J, Lisman JE. Oscillations in the alpha band $(9-12 \mathrm{~Hz})$ increase with memory load during retention in a short-term memory task. Cereb Cortex 12: 877-882, 2002.

Kaufman EF, Rosenquist AC. Efferent projections of the thalamic intralaminar nuclei in the cat. Brain Res 335: 257-279, 1985.

Klier EM, Angelaki DE. Spatial updating and the maintenance of visual constancy. Neuroscience 156: 801-818, 2008.

Klier EM, Hess BJ, Angelaki DE. Human visuospatial updating after passive translations in three-dimensional space. J Neurophysiol 99: 1799-1809, 2008.

Kovács G, Raabe M, Greenlee MW. Neural correlates of visually induced self-motion illusion in depth. Cereb Cortex 18: 1779-1787, 2008.

Kusunoki M, Goldberg ME. The time course of perisaccadic receptive field shifts in the lateral intraparietal area of the monkey. $J$ Neurophysiol 89: 1519-1527, 2003.

Li N, Angelaki DE. Updating visual space during motion in depth. Neuron 48: 149-158, 2005.

Li N, Wei M, Angelaki DE. Primate memory saccade amplitude after intervened motion depends on target distance. J Neurophysiol 94: 722-733, 2005.

Lopez C, Blanke O, Mast FW. The human vestibular cortex revealed by coordinate-based activation likelihood estimation meta-analysis. Neuroscience 212: 159-179, 2012.

Maris E, Oostenveld R. Nonparametric statistical testing of EEG- and MEG-data. J Neurosci Methods 164: 177-190, 2007.

Medendorp W. Spatial constancy mechanisms in motor control. Philos Trans $R$ Soc Lond B Biol Sci 366: 476-491, 2011.

Medendorp WP, Goltz HC, Vilis T, Crawford JD. Gaze-centered updating of visual space in human parietal cortex. J Neurosci 23: 6209-6214, 2003a.

Medendorp WP, Tweed DB, Crawford JD. Motion parallax is computed in the updating of human spatial memory. J Neurosci 23: 8135-8142, $2003 \mathrm{~b}$.

Medendorp WP, Van Asselt S, Gielen CC. Pointing to remembered visual targets after active one-step self-displacements within reaching space. Exp Brain Res 125: 50-60, 1999.

Meng H, May PJ, Dickman JD, Angelaki DE. Vestibular signals in primate thalamus: properties and origins. J Neurosci 27: 13590-13602, 2007.

Merriam EP, Genovese CR, Colby CL. Spatial updating in human parietal cortex. Neuron 39: 361-373, 2003.

Morris AP, Chambers CD, Mattingley JB. Parietal stimulation destabilizes spatial updating across saccadic eye movements. Proc Natl Acad Sci USA 104: 9069-9074, 2007.

Oostenveld R, Fries P, Maris E, Schoffelen JM. FieldTrip: open source software for advanced analysis of MEG, EEG, and invasive electrophysiological data. Comput Intell Neurosci 2011: 156869, 2011.

Osipova D, Hermes D, Jensen O. Gamma power is phase-locked to posterior alpha activity. PLoS One 3: e3990, 2008.

Prevosto V, Graf W, Ugolini G. Posterior parietal cortex areas MIP and LIPV receive eye position and velocity inputs via ascending preposito-thalamocortical pathways. Eur J Neurosci 30: 1151-1161, 2009.

Raghavachari S, Lisman JE, Tully M, Madsen JR, Bromfield EB, Kahana MJ. Theta oscillations in human cortex during a working-memory task: evidence for local generators. J Neurophysiol 95: 1630-1638, 2006.

Roberts BM, Hsieh LT, Ranganath C. Oscillatory activity during maintenance of spatial and temporal information in working memory. Neuropsychologia 51: 349-357, 2013.

Roux F, Uhlhaas PJ. Working memory and neural oscillations: alpha-gamma versus theta-gamma codes for distinct WM information? Trends Cogn Sci 18: 16-25, 2014.

Salazar RF, Dotson NM, Bressler SL, Gray CM. Content-specific frontoparietal synchronization during visual working memory. Science 338: $1097-$ 1100, 2012.

Sauseng P, Klimesch W, Doppelmayr M, Pecherstorfer T, Freunberger R, Hanslmayr S. EEG alpha synchronization and functional coupling during top-down processing in a working memory task. Hum Brain Mapp 26: $148-155,2005$. 
Sauseng P, Klimesch W, Heise KF, Gruber WR, Holz E, Karim AA, Glennon M, Gerloff C, Birbaumer N, Hummel FC. Brain oscillatory substrates of visual short-term memory capacity. Curr Biol 19: 1846-1852, 2009.

Schlag J, Schlag-Rey M. Through the eye, slowly: delays and localization errors in the visual system. Nat Rev Neurosci 3: 191-215, 2002.

Shinder ME, Taube JS. Differentiating ascending vestibular pathways to the cortex involved in spatial cognition. J Vestib Res 20: 3-23, 2010.

Sommer MA, Wurtz RH. Influence of the thalamus on spatial visual processing in frontal cortex. Nature 444: 374-377, 2006.

Suzuki M, Kitano H, Ito R, Kitanishi T, Yazawa Y, Ogawa T, Shiino A, Kitajima K. Cortical and subcortical vestibular response to caloric stimulation detected by functional magnetic resonance imaging. Brain Res Cogn Brain Res 12: 441-449, 2001.

Tremblay L, Kennedy A, Paleressompoulle D, Borel L, Mouchnino L, Blouin J. Biases in the perception of self-motion during whole-body acceleration and deceleration. Front Integr Neurosci 7: 90, 2013.
VanRullen R. Visual attention: a rhythmic process? Curr Biol 23: R1110R1112, 2013

Van Der Werf J, Buchholz VN, Jensen O, Medendorp WP. Reorganization of oscillatory activity in human parietal cortex during spatial updating. Cereb Cortex 23: 508-519, 2013.

Van Pelt S, Medendorp WP. Gaze-centered updating of remembered visual space during active whole-body translations. J Neurophysiol 97: 12091220, 2007.

von Helmholtz H. Handbuch der Physiologischen Optik. Hamburg, Germany: Voss, 1867.

Wolbers T, Hegarty M, Büchel C, Loomis JM. Spatial updating: how the brain keeps track of changing object locations during observer motion. Nat Neurosci 11: 1223-1230, 2008.

Worden MS, Foxe JJ, Wang N, Simpson GV. Anticipatory biasing of visuospatial attention indexed by retinotopically specific alpha-band electroencephalography increases over occipital cortex. J Neurosci 20: RC63, 2000.

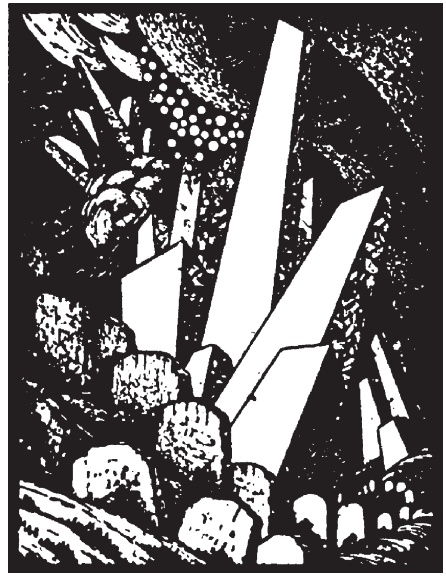

